\title{
Serum lysyl oxidase-like 2 levels and idiopathic pulmonary fibrosis disease progression
}

\author{
Jason W. Chien', Thomas J. Richards², Kevin F. Gibson², Yingze Zhang², \\ Kathleen O. Lindell ${ }^{2}$, Lixin Shao ${ }^{3}$, Susan K. Lyman ${ }^{3}$, Joanne I. Adamkewicz ${ }^{3}$, \\ Victoria Smith ${ }^{3}$, Naftali Kaminski ${ }^{2,4}$ and Thomas O'Riordan ${ }^{1}$
}

Affiliations: ${ }^{1}$ Gilead Sciences, Inc., Seattle, WA, ${ }^{2}$ The Dorothy P. and Richard P. Simmons Center for Interstitial Lung Disease, University of Pittsburgh, Pittsburgh, PA, ${ }^{3}$ Gilead Sciences, Inc., Foster City, CA, and 4 Pulmonary, Critical Care and Sleep Medicine, Yale School of Medicine, New Haven, CT, USA.

Correspondence: J.W. Chien, Gilead Sciences Inc., 199 E. Blaine St., Seattle, WA 98102, USA.

E-mail: jason.chiendagilead.com

ABSTRACT We evaluated whether lysyl oxidase-like 2 (LOXL2), which promotes cross-linking of collagen in pathological stroma, was detectable in serum from idiopathic pulmonary fibrosis (IPF) patients, and assessed its relationship with IPF disease progression.

Patients from the ARTEMIS-IPF $(\mathrm{n}=69)$ and the Genomic and Proteomic Analysis of Disease Progression in IPF (GAP) $(n=104)$ studies were analysed. Baseline serum LOXL2 (sLOXL2) levels were compared with baseline clinical and physiological surrogates of disease severity, and the association with IPF disease progression was assessed using a classification and regression tree (CART) method.

sLOXL2 correlated weakly with forced vital capacity and carbon monoxide diffusion capacity $(\mathrm{r}-0.24-0.05)$ in both cohorts. CART-determined thresholds were similar: ARTEMIS-IPF $800 \mathrm{pg} \cdot \mathrm{mL}^{-1}$ and GAP $700 \mathrm{pg} \cdot \mathrm{mL}^{-1}$. In ARTEMIS-IPF, higher sLOXL2 $\left(>800 \mathrm{pg} \cdot \mathrm{mL}^{-1}\right)$ was associated with increased risk for disease progression (hazard ratio (HR) 5.41, 95\% CI 1.65-17.73). Among GAP subjects with baseline spirometric data $(n=70)$, higher sLOXL2 levels $\left(>700 \mathrm{pg} \cdot \mathrm{mL}^{-1}\right)$ were associated with more disease progression events (HR 1.78, 95\% CI 1.01-3.11). Among all GAP subjects, higher sLOXL2 levels were associated with increased risk for mortality (HR 2.28, 95\% CI 1.18-4.38).

These results suggest that higher sLOXL2 levels are associated with increased risk for IPF disease progression. However, due to multiple limitations, these results require validation.

@ERSpublications

Higher baseline sLOXL2 levels: no correlation with IPF severity, but association with disease progression http://ow.ly/sEbGW

For editorial comments see page 1233 .

This article has supplementary material available from www.erj.ersjournals.com

Received: May 082013 | Accepted after revision: Sept 192013 | First published online: Oct 312013

Clinical trial: This study is registered at www.ClinicalTrials.gov with identifier number NCT00768300 and NCT 00373841.

Support statement: This work was funded by Gilead Sciences Inc. and a National Institutes of Health grant (grant number P50HL084932).

Conflict of interest: Disclosures can be found alongside the online version of this article at www.erj.ersjournals.com

Copyright @ERS 2014 


\section{Introduction}

Idiopathic pulmonary fibrosis (IPF) is a progressive and ultimately fatal disease of the lungs involving airway epithelial cell damage, fibroblast activation and proliferation, and excessive deposition of collagen and other extracellular matrix (ECM) components [1]. Increased deposition of fibrillar collagens I and III is apparent [2], along with changes in the abundance and localisation of collagens V, XII and XIV, which help to organise and stabilise the growing fibrils [3,4]. These modifications of ECM composition and organisation alter the biomechanical properties of the lung parenchyma and increase local tension, which is critical in IPF disease pathogenesis [5].

One important driver of matrix tension is lysyl oxidase-like 2 (LOXL2), an enzyme that catalyses covalent cross-linking of ECM molecules, including fibrillar collagens. Activated fibroblasts secrete LOXL2 along with increased amounts of collagen [6]. This pathological remodelling of the ECM probably results in the release of latent transforming growth factor (TGF)- $\beta$ from the latency-associated peptide complex sequestered in the ECM $[7,8]$, driving a feed-forward loop of cell activation, LOXL2 and collagen secretion, and matrix stiffening.

LOXL2 protein expression is observed in the fibroblastic foci and collagenous regions of diseased IPF lung tissue, with relatively minor expression in healthy lung tissue [9]. LOXL2 has also been localised to the active disease interface in liver fibrosis [9], and is considered a "core" driver in fibrosis [10]. As LOXL2 is associated with areas of active fibrogenesis in diseased tissues, we hypothesised that serum levels of LOXL2 (sLOXL2) would be elevated in IPF patients. We developed an immunoassay capable of relative quantification of the circulating levels of LOXL2 protein, and tested serum samples collected from two independent cohorts of well-characterised IPF patients. The main objective of this study was to evaluate whether sLOXL2 levels at baseline are associated with measures of IPF disease severity and the risk for IPF progression. Preliminary results of this study have been previously reported at several international meetings $[11,12]$.

\section{Methods}

This study was conducted using serum and clinical data collected from subjects participating in two independent clinical studies: ARTEMIS-IPF and the Genomic and Proteomic Analysis of Disease Progression in IPF (GAP) study. All serum and data were collected prospectively, but analysed retrospectively. All analyses were approved by the institutional review boards of each clinical site.

\section{Study populations and serum collection}

ARTEMIS-IPF (registered at www.ClinicalTrials.gov with identifier NCT00768300) was a multicentre, randomised, double-blind, placebo-controlled, event-driven trial that evaluated whether ambrisentan, a selective antagonist of the endothelin type A receptor approved for treatment of pulmonary arterial hypertension, could prevent IPF disease progression [13]. Baseline clinical and physiological data included forced vital capacity (FVC) \% predicted (\% pred), diffusing capacity of the lung for carbon monoxide (DLCO) \% pred, 6-min walking distance (6MWD), mean pulmonary arterial pressure (PAP), the St George's Respiratory Questionnaire (SGRQ) score and the Transition Dyspnoea Index (TDI) score. The PAP was obtained via a right heart catheterisation, which was required of all study subjects at baseline. The composite physiological index (CPI) was calculated using the baseline FVC and DLCO according to a previously published formula [14].

The GAP study (registered at www.ClinicalTrials.gov with identifier NCT00373841) is a prospective observational study that recruits subjects at the University of Pittsburgh Dorothy P. and Richard P. Simmons Center for Interstitial Lung Disease (Pittsburgh, PA, USA). Recruitment for this cohort began in October 2005. The database was locked for the current analysis on December 1, 2011. The study subjects were required to be 1) $\geqslant 18$ years of age; 2) have a diagnosis of IPF according to American Thoracic Society/ European Respiratory Society guidelines [15]; and 3) able to receive all ongoing care and follow-up at the clinical facility. Clinical data collected between 3 months prior to and 2 weeks after study visit 1 were included as part of the baseline assessment, which included, but was not limited to, lung function testing.

For ARTEMIS-IPF, baseline serum was collected prior to randomisation at select clinical sites in the USA. These sites had the necessary infrastructure and support staff for collection of these samples as specified according to the laboratory protocol. For the GAP cohort, baseline serum samples were collected at enrolment study visit, then every 4-6 months thereafter during routine clinic visits. After collection, serum samples were aliquoted and frozen at $-80^{\circ} \mathrm{C}$; all serum samples analysed for this study were maintained at $-80^{\circ} \mathrm{C}$ until testing. 
Healthy donor serum

sLOXL2 levels were analysed in samples from self-reported "healthy" adult donors purchased from Bioreclamation Inc. (Hicksville, NY, USA) and Cureline Inc. (South San Francisco, CA, USA). Donors were seronegative for hepatitis $\mathrm{B}$, hepatitis $\mathrm{C}$ and HIV infection.

\section{Clinical end-points}

The same pre-specified clinical end-points used in the ARTEMIS-IPF clinical trial were used for the sLOXL2 analysis. The primary end-point was time to the first IPF disease progression event, a composite end-point composed of mortality from any cause, adjudicated respiratory hospitalisations, or a categorical decrease in lung function (defined as either a $\geqslant 10 \%$ decrease in FVC with a $\geqslant 5 \%$ decrease in the DLCO or a $\geqslant 15 \%$ decrease in DLCO with a $\geqslant 5 \%$ decrease in FVC). For the GAP cohort, the ARTEMIS-IPF disease progression definition was applied to the subset of subjects with baseline spirometric measurements $(\mathrm{n}=70)$, but without including respiratory hospitalisation events. Hospitalisations were not tracked in the GAP cohort because these patients were referred from other healthcare programmes, where they were hospitalised when necessary. All-cause mortality was evaluated among all subjects with baseline sLOXL2 available for analysis $(n=104)$. If lung transplantation occurred during the follow-up period, it was considered a mortality event.

\section{Antibody generation and sLOXL2 immunoassay}

Details of the antibody generation methods and sLOXL2 immunoassay procedure and validation can be found in the online supplementary material. Briefly, two anti-LOXL2 antibodies, one rabbit polyclonal and one mouse monoclonal, were generated against full-length purified LOXL2 protein. A sandwich immunoassay using an electrochemiluminescent platform (Meso Scale Discovery, Rockville, MD, USA) was developed using these two anti-LOXL2 antibodies. Purified recombinant human LOXL2 protein (R\&D Systems, Minneapolis, MN, USA) was used as the calibrator, allowing for relative quantification of unknown samples.

\section{Statistical analysis}

A staged approach was used to analyse the ARTEMIS-IPF and GAP cohorts. The first stage consisted of nine association analyses in the ARTEMIS-IPF cohort; eight focused on baseline characteristics and one focused on the disease progression end-point. A Bonferroni correction for multiple comparisons required the $\alpha$ threshold to be set at 0.006 . The second stage was a hypothesis driven analysis of the GAP cohort, where the primary analysis evaluated whether the association between baseline sLOXL2 levels with IPF disease progression can be replicated at the $\alpha$-threshold of 0.05 . All testing with the components of the disease progression end-point was considered exploratory, given each of these end-points was a component of the disease progression end-point.

All analyses were performed using SAS statistical software, version 9.1 (SAS Institute Inc., Cary, NC, USA). Patient samples with sLOXL2 levels below the assay lower limit of detection (LLOD) $\left(180 \mathrm{pg} \cdot \mathrm{mL}^{-1}\right)$ were assigned the value LLOD/2 $\left(90 \mathrm{pg} \cdot \mathrm{mL}^{-1}\right)$ for purposes of analysis, as recommended for left-censored data [16]. Pearson's correlation coefficient was used to determine correlations between sLOXL2 levels and demographic and clinical variables. A classification and regression trees (CART) method, with IPF disease progression status (binary) as response variable and baseline sLOXL2 level as dependent variable, was used to select the "low" versus "high" sLOXL2 subjects to evaluate the association between the IPF disease progression and baseline sLOXL2. CART was applied in an unsupervised fashion independently to each cohort. Differences in probability of developing an end-point for high and low sLOXL2 groups were calculated using cumulative incidence curves. The association between an end-point and sLOXL2 levels was assessed independently for each cohort using Cox proportional hazard modelling. A stepwise selection method was used to identify baseline variables that were associated with the end-point of interest at the $p<0.05$ threshold. Treatment assignment was forced into the models for the ARTEMIS-IPF analyses. Follow-up for ARTEMIS-IPF was for the full duration of the study. GAP cohort subjects were followed until death, lung transplantation or censored at 24-months after enrolment. Statistical significance was defined as a two-sided p-value of $<0.05$.

\section{Results}

\section{LOXL2 immunoassay and characterisation of healthy donors}

Development of the LOXL2 immunoassay was conducted using calibrator samples generated by adding recombinant full-length human LOXL2 protein into serum from normal healthy donors lacking detectable LOXL2 levels, as described in the online supplementary material. Incurred patient samples were used for validation. Assay performance characteristics for serum samples are shown in table 1; assay performance for EDTA plasma samples was similar (data not shown). 
TABLE 1 Serum lysyl oxidase-like 2 (LOXL2) immunoassay performance characteristics

Assay characteristic
Accuracy relative error
Intra-assay precision coefficient of variation
Inter-assay precision coefficient of variation
LLOD
LLOQ
Upper limit of quantitation

Result

$<15 \%$
$5 \%$
$25 \%$
$180 \mathrm{pg} \cdot \mathrm{mL}^{-1}$
$440 \mathrm{pg} \cdot \mathrm{mL}^{-1}$
limit not determined;
calibrator standard
as $132118 \mathrm{pg} \cdot \mathrm{mL}^{-1}$

Accuracy was determined using pooled serum (from healthy individuals) spiked with recombinant LOXL2 protein. Intra-assay and inter-assay precision were determined using incurred samples. Lower limit of detection (LLOD) is the mean $+2.5 \times \mathrm{SD}$ of the blank wells. Lower limit of quantitation (LLOQ) is the lowest calibrator standard with relative error $<30 \%$ and coefficient of variation $<30 \%$.

Samples from self-reported healthy adult donors $(n=101)$ unrelated to the IPF study participants were tested for sLOXL2 levels. The mean \pm SD age of these donors was $50.3 \pm 14.3$ years and $60 \%$ were males. There was no association between age $(\mathrm{p}=0.36)$ with sLOXL2 levels. $90 \%$ (91 donors) had sLOXL2 levels less than LLOD and 2\% (two donors) had sLOXL2 levels $<$ lower limit of quantitation.

\section{sLOXL2 in the ARTEMIS-IPF study}

Among the 492 subjects in the intent-to-treat cohort, 69 (14\%) subjects (49 treated with ambrisentan) had baseline serum samples collected and available for analysis. The mean follow-up time for these subjects was 245 days. There were nine deaths during this follow-up period for this subgroup with serum available. These subjects, in comparison with subjects without baseline serum, had similar baseline demographic characteristics and IPF severity, as reflected by \% predicted FVC and DLCO, the 6MWD, mean PAP, CPI, SGRQ score and the TDI score (table 2).

The median baseline sLOXL2 level for all 69 subjects was $304 \mathrm{pg} \cdot \mathrm{mL}^{-1}$ (fig. 1) (interquartile range (IQR) 144-738 $\mathrm{pg} \cdot \mathrm{mL}^{-1}$; minimum $<180 \mathrm{pg} \cdot \mathrm{mL}^{-1}$ (below LLOD), maximum $5389 \mathrm{pg} \cdot \mathrm{mL}^{-1}$ ); the median baseline

TABLE 2 Baseline characteristics and measurements of subjects in the ARTEMIS-IPF study and Genomic and Proteomic Analysis of Disease Progression in Idiopathic Pulmonary Fibrosis (GAP) study cohort

\begin{tabular}{lccc} 
Baseline characteristics and & \multicolumn{2}{c}{ ARTEMIS-IPF } & \multirow{2}{*}{ GAP cohort } \\
\cline { 2 - 3 } measurements & No serum & Serum & \\
\cline { 2 - 3 } Subjects & 423 & 69 & 104 \\
Age years & $65.9 \pm 7.3$ & $66.2 \pm 7.0$ & $66.7 \pm 8.9$ \\
Males & $303(72)$ & $52(75)$ & $73(70)$ \\
FVC \% predicted & $69.0 \pm 13.5$ & $69.8 \pm 12.1$ & $66.1 \pm 17.7^{\#}$ \\
DLCo \% predicted & $43.4 \pm 14.1$ & $42.1 \pm 11.1$ & $47.8 \pm 18.0^{\#}$ \\
6MWD m & $416.2 \pm 120.1$ & $398.6 \pm 115.8$ & $\mathrm{NA}$ \\
PAP mmHg & $20.4 \pm 7.1$ & $20.4 \pm 5.9$ & $\mathrm{NA}$ \\
CPI score & $52.2 \pm 10.8$ & $52.5 \pm 8.9$ & $51.0 \pm 13.0$ \\
SGRQ score & $39.4 \pm 19.5$ & $38.0 \pm 18.4$ & $\mathrm{NA}$ \\
TDI score & $7.3 \pm 2.4$ & $7.5 \pm 2.4$ & $\mathrm{NA}$ \\
Follow-up days median \pm SD & $237 \pm 143$ & $245 \pm 177$ & $455 \pm 340$ \\
Deaths & $23(5)$ & $9(13)$ & $43(41)^{\bullet}$ \\
\hline
\end{tabular}

Data are presented as $n$, mean \pm SD or $n(\%)$, unless otherwise stated. FVC: forced vital capacity; DLCo: diffusing capacity of the lung for carbon monoxide; 6MWD: 6-min walking distance; PAP: pulmonary arterial pressure; $\mathrm{CPI}$ : composite physiological index; SGRQ: St George's Respiratory Questionnaire; TDI: transition dyspnoea index; NA: not applicable. \#: only $70(67 \%)$ subjects had baseline pulmonary function data available; ^: censored at 24 months after enrolment, lung transplant $(n=17)$ was treated as a mortality event. 


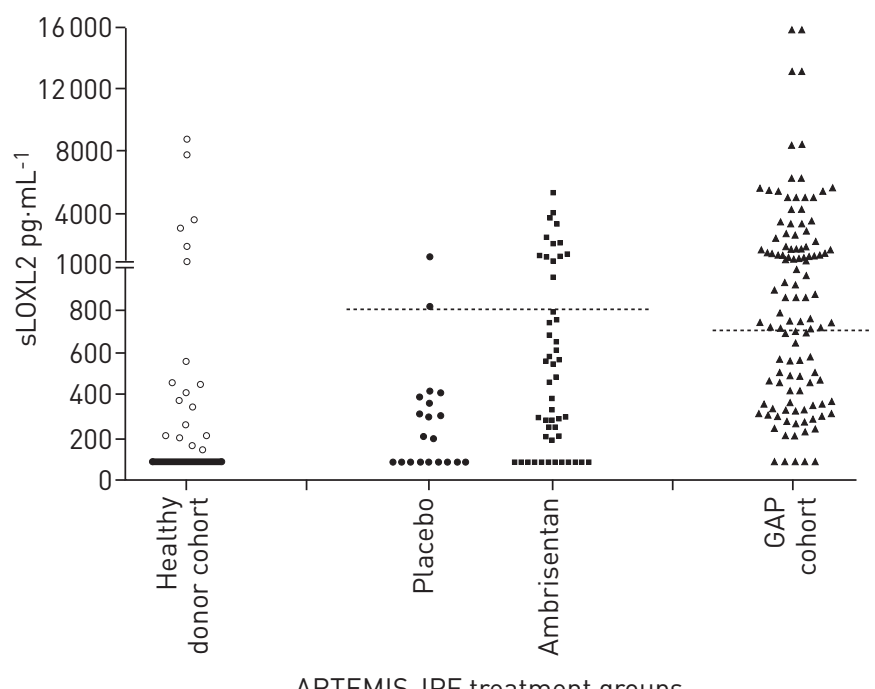

FIGURE 1 Distribution of serum lysyl oxidase-like 2 (sLOXL2) among subjects treated with ambrisentan and placebo in ARTEMIS-IPF and subjects in the Genomic and Proteomic Analysis of Disease Progression in Idiopathic Pulmonary Fibrosis (GAP) study cohort. The dotted lines represent thresholds used for dichotomisation of subjects in to "low" and "high" sLOXL2 groups in the ARTEMIS-IPF $\left(800 \mathrm{pg} \cdot \mathrm{mL}^{-1}\right)$ and GAP cohorts $\left(700 \mathrm{pg} \cdot \mathrm{mL}^{-1}\right)$.

sLOXL2 level for placebo- and ambrisentan-treated subjects was $206.1 \mathrm{pg} \cdot \mathrm{mL}^{-1}$ and $360.6 \mathrm{pg} \cdot \mathrm{mL}^{-1}$ respectively. Correlation was weak between sLOXL2 and baseline demographic and physiological measures of IPF severity (correlation coefficients were as follows: age 0.31 ; FVC - 0.24 ; DLCO - 0.13 ; 6MWD -0.04; PAP 0.17; CPI 0.14; SGRQ 0.12; and TDI -0.18). CART analysis of baseline sLOXL2 levels identified $800 \mathrm{pg} \cdot \mathrm{mL}^{-1}$ as the optimal threshold for classifying the IPF disease progression risk in this cohort; $36(67 \%)$ and 18 $(33 \%)$ ambrisentan-treated subjects were subgrouped as having "low" ( $\left.\leqslant 800 \mathrm{pg} \cdot \mathrm{mL}^{-1}\right)$ versus "high" $\left(>800 \mathrm{pg} \cdot \mathrm{mL}^{-1}\right)$ baseline sLOXL2 levels, respectively; $13(87 \%)$ and two (13\%) placebo treated subjects were subgrouped as having "low" ( $\left.\leqslant 800 \mathrm{pg} \cdot \mathrm{mL}^{-1}\right)$ versus "high" $\left(>800 \mathrm{pg} \cdot \mathrm{mL}^{-1}\right)$ baseline sLOXL2 levels, respectively.

Presence of a high baseline sLOXL2 level was associated with higher probability for experiencing IPF disease progression events (fig. 2a). This association was consistently observed for all three components of the disease progression end-point: lung function decline (fig. 2b), respiratory hospitalisations (fig. 2c) and mortality (fig. 2d). Cox proportional hazard modelling (table 3) revealed that a high baseline sLOXL2 level was associated with a 5.4-fold increase in risk for IPF disease progression $(p=0.005)$, a 7.6 -fold increase in risk for lung function decline $(\mathrm{p}=0.031)$, and a 5.4 -fold increase in risk for respiratory hospitalisations $(p=0.029)$. Treatment assignment was included in each of these models, as were 6MWD and CPI scores, which were the only baseline variables found to remain significant using a stepwise approach.

\section{sLOXL2 in the GAP cohort}

Overall, 120 subjects were enrolled into the GAP cohort. Among these, 105 had serum samples available for analysis, out of which one subject did not have adequate clinical data. Of the remaining 104 subjects, 70 had baseline spirometry values. In comparison with ARTEMIS-IPF, GAP subjects tended to have lower baseline FVC but slightly higher DLCO (table 2). The mean follow-up time was also longer at 455 days. All data were censored at 2 years after enrolment for the remaining analyses. During this period, there were 26 deaths and 17 lung transplants in this cohort.

The median baseline sLOXL2 level for the 104 subjects was $716 \mathrm{pg} \cdot \mathrm{mL}^{-1}$ (fig. 1) (IQR $<180-1447 \mathrm{pg} \cdot \mathrm{mL}^{-1}$ (lower value below LLOD); minimum $<180 \mathrm{pg} \cdot \mathrm{mL}^{-1}$ (below LLOD), maximum $15708 \mathrm{pg} \cdot \mathrm{mL}^{-1}$ ). In the subset of subjects with baseline spirometry data available, correlation between the baseline sLOXL2 levels and lung function was weak (correlation coefficients were as follows: age -0.16; FVC -0.01; DLCO -0.28; and CPI 0.24). CART analysis identified $700 \mathrm{pg} \cdot \mathrm{mL}^{-1}$ as the optimal threshold for classifying the cohort for IPF disease progression risk; $46(44 \%)$ subjects were subgrouped as having "low" ( $\left.\leqslant 700 \mathrm{pg} \cdot \mathrm{mL}^{-1}\right) \mathrm{sLOXL} 2,58$ $(56 \%)$ subjects were subgrouped as having "high" $\left(>700 \mathrm{pg} \cdot \mathrm{mL}^{-1}\right) \mathrm{sLOXL} 2$.

Among the 70 subjects with baseline spirometric measurements, presence of a high baseline sLOXL2 level was associated with significantly more IPF disease progression events $(p=0.042)$ (fig. 3a). Cox proportional hazard model (table 3) revealed that high baseline sLOXL2 level was associated with a 1.8 -fold increase in 

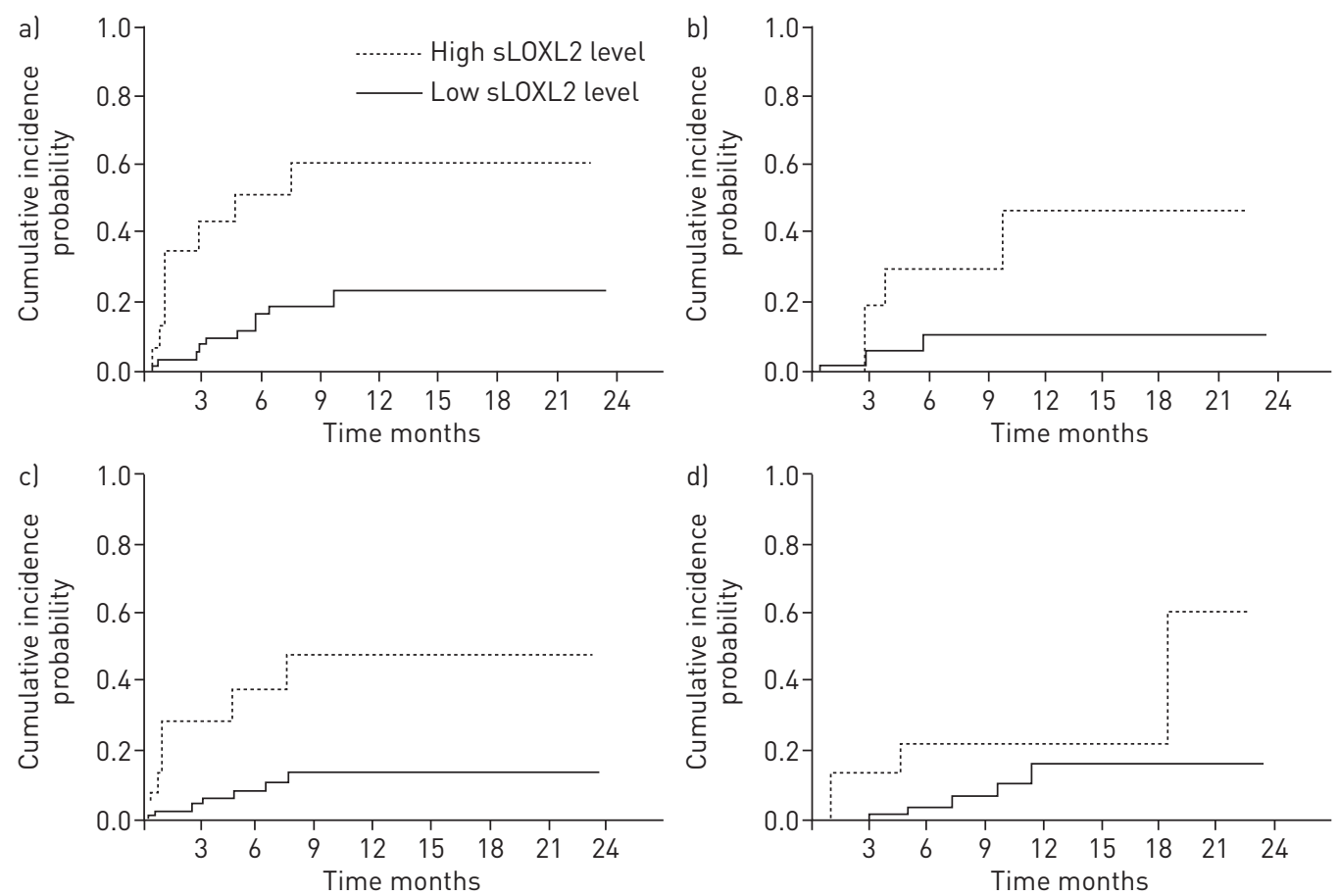

FIGURE 2 Cumulative incidence curves comparing low $\left(\leqslant 800 \mathrm{pg} \cdot \mathrm{mL}^{-1}\right)$ and high $\left(>800 \mathrm{pg} \cdot \mathrm{mL}^{-1}\right)$ serum lysyl oxidaselike 2 (sLOXL2) levels in ARTEMIS-IPF for a) disease progression and its components: b) lung function decline, c) respiratory hospitalisations and d) death.

risk for disease progression $(\mathrm{p}=0.045)$. Although lung function decline $(\mathrm{p}=0.334)$ and mortality $(\mathrm{p}=0.159)$, when considered as individual components, did not meet the $0.05 \mathrm{p}$-value threshold, presence of a high baseline sLOXL2 level was associated with a higher probability of experiencing one of these events (figs $3 \mathrm{~b}$ and c). Mortality analysis of all 104 subjects in the GAP cohort revealed that high baseline sLOXL2 levels were associated with more deaths $(\mathrm{p}=0.017)$ (fig. $3 \mathrm{~d})$, and a 2.2 -fold increase in risk for death $(\mathrm{p}=0.014)$ (table 3). Each of these statistical models included sex and age as covariates, which were the only baseline variables found to remain significant using a stepwise approach.

TABLE 3 Hazard ratios for end-points in the ARTEMIS-IPF study according to low versus high baseline serum lysyl oxidase-like 2 (sLOXL2)

End-points

Event rate

HR $(95 \%$ Cl) for high sLOXL2

p-value

Low sLOXL2 High sLOXL2

\section{ARTEMIS-IPF}

Disease progression

Lung function decline

Respiratory hospitalisation

Mortality

GAP

Disease progression

Lung function decline

Mortality

Mortality all subjects

$\begin{array}{cc}10 / 54(19) & 8 / 15(53) \\ 5 / 54(9) & 4 / 15(27) \\ 6 / 54(11) & 6 / 15(40) \\ 5 / 54(9) & 4 / 15(27) \\ & \\ 22 / 35(63) & 28 / 35(80) \\ 13 / 35(37) & 16 / 35(46) \\ 10 / 35(29) & 16 / 35(46) \\ 13 / 46(28) & 30 / 58(52)\end{array}$

$\begin{array}{ll}5.41(1.65-17.73) & 0.005 \\ 7.64(1.21-48.25) & 0.031 \\ 5.35(1.19-24.00) & 0.029 \\ 1.87(0.28-12.45) & 0.517 \\ & \\ 1.78(1.01-3.11) & 0.045 \\ 1.43(0.69-2.98) & 0.337 \\ 1.77(0.80-3.89) & 0.159 \\ 2.28(1.18-4.38) & 0.014\end{array}$

Data are presented as n/N (\%), unless otherwise stated. GAP: Genomic and Proteomic Analysis of Disease Progression in Idiopathic Pulmonary Fibrosis study. " : each model for ARTEMIS-IPF included treatment assignment, 6-min walking distance and composite physiological index as covariates. ": the first three models (disease progression, lung function decline and all-cause mortality) included only subjects with baseline spirometry $(n=70)$. These models included no covariates. The second mortality model that included all subjects ( $n=104)$ included sex as a covariate. 

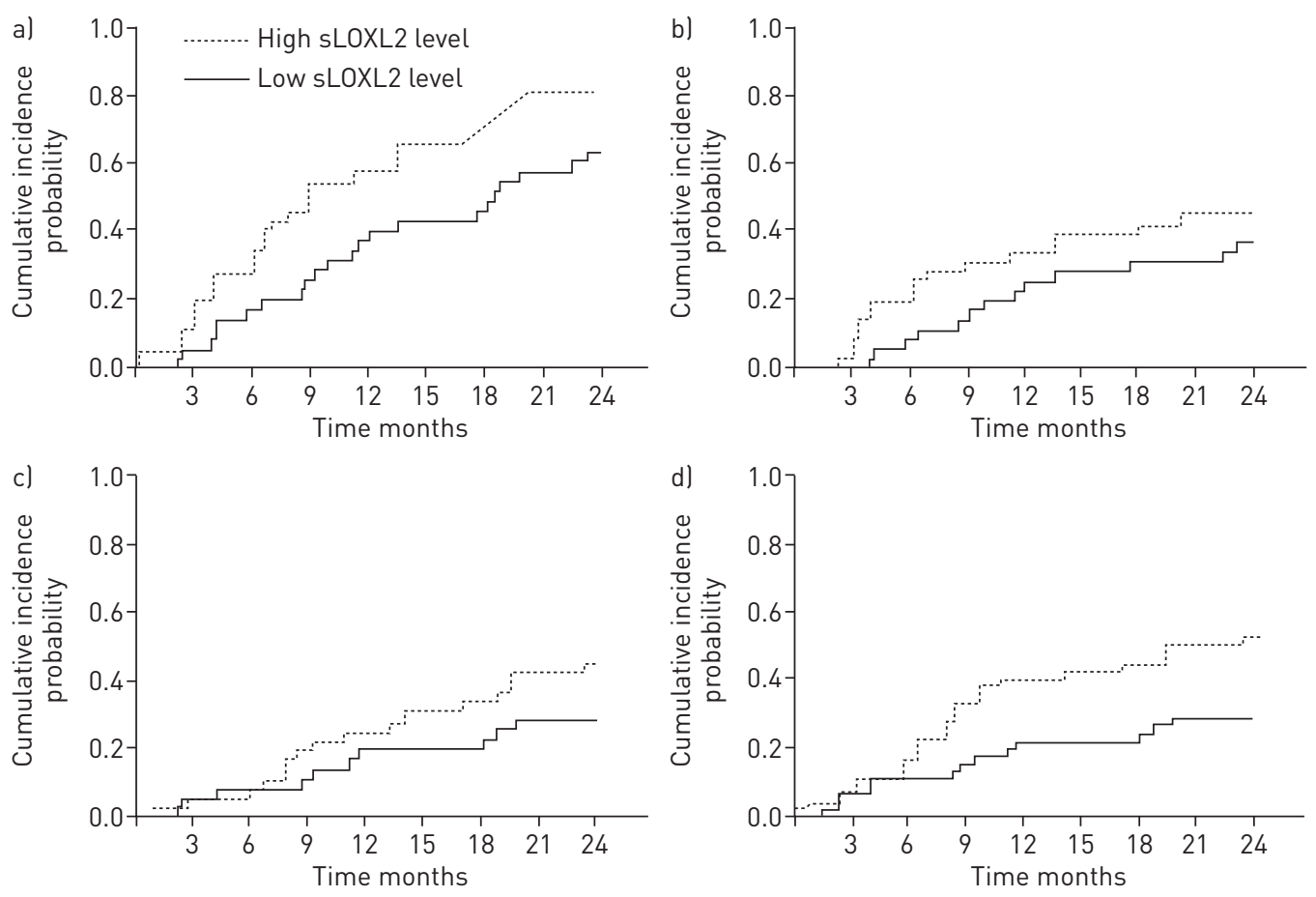

FIGURE 3 Cumulative incidence curves comparing low $\left(\leqslant 700 \mathrm{pg} \cdot \mathrm{mL}^{-1}\right)$ and high $\left(>700 \mathrm{pg} \cdot \mathrm{mL}^{-1}\right)$ serum lysyl oxidaselike 2 (sLOXL2) levels for a) disease progression and its components: b) lung function decline, c) death among Genomic and Proteomic Analysis of Disease Progression in Idiopathic Pulmonary Fibrosis (GAP) study subjects with $(\mathrm{n}=70)$ baseline spirometric values and d) death among GAP study subjects without $(\mathrm{n}=104)$ baseline spirometric values.

\section{Discussion}

LOXL2 is directly involved in disease-related fibrogenesis [9]: it promotes cross-linking of fibrillar collagen I, a major component of desmoplastic stroma and fibrosis, and plays a crucial role in matrix remodelling, fibrogenesis and formation of pathological stroma. In this study, we demonstrated that LOXL2 can be detected in circulation and, among patients with IPF, we found a novel association between sLOXL2 levels and IPF disease progression. We also found that baseline sLOXL2 levels were not significantly correlated with typical clinical measures of IPF disease severity (e.g. FVC and DLCO). Given the current understanding of the role of LOXL2 in fibrosis, these findings suggest that circulating sLOXL2 levels may be reflective of IPF disease activity, rather than severity.

Although these results represent an association and not a direct causal relationship between sLOXL2 levels and IPF outcomes, there are several reasons to believe that sLOXL2 is worthy of further investigation as a potential biomarker for IPF disease activity. First, there is strong biological evidence for a causal relationship between high sLOXL2 levels and poor IPF outcomes. We demonstrated previously that LOXL2 is expressed in murine models of fibrotic disease (e.g. lung fibrosis and liver fibrosis), and can be inhibited by treatment with an anti-LOXL2 antibody [9]. In a therapeutic murine model of pulmonary fibrosis, GS-607601 (formerly known as AB0023), a mouse monoclonal antibody that inhibits LOXL2, was able to reduce LOXL2 levels in the lung, reduce factors that reflect fibrotic activity (e.g. number of $\alpha$ SMA-positive fibroblasts, stromal cell-derived factor- $\alpha 1$, TGF- $\beta 1$, endothelin- 1 and phospho-SMAD2) and reduce the extent of fibrosis. In humans with hepatitis B- or C-associated liver fibrosis, high sLOXL2 levels have also been demonstrated to be associated with higher Ishak fibrosis score, and sLOXL2 levels decline with antihepatitis B therapy $[17,18]$. Thus, both nonhuman and human data support the possibility that sLOXL2 is reflective of the extent of fibrotic disease activity.

Secondly, sLOXL2 was consistently associated with disease progression and its components in both IPF cohorts. In ARTEMIS-IPF, the lung function decline and respiratory hospitalisations components of the disease progression end-point were most strongly associated with high sLOXL2 levels. The association with mortality risk also trended in the same direction, although it did not meet the $0.05 \mathrm{p}$-value threshold. In the GAP cohort, both the lung function decline and mortality risks met the $0.05 \mathrm{p}$-value threshold. Significantly, the mortality risk associated with high sLOXL2 levels in the GAP cohort was of similar magnitude to that observed in ARTEMIS-IPF, suggesting that if the mortality event rate were higher in ARTEMIS-IPF, the $0.05 \mathrm{p}$-value threshold would have probably been achieved. 
Thirdly, these associations were observed in two independent populations that are quite different on multiple levels, setting our analysis up as a sensitivity analysis for the relationship between baseline sLOXL2 and IPF clinical outcomes. The ARTEMIS-IPF population was recruited within the context of a wellcontrolled multicentre clinical trial in which the inclusion and exclusion criteria were designed to identify subjects with mild IPF; subjects with $>5 \%$ honeycombing on high-resolution computed tomography were excluded [13]. The GAP cohort is an observational cohort referred for evaluation at an interstitial lung disease programme located in a tertiary institution where patients are more likely to have advanced IPF. Although the FVC and DLCO were similar between these two cohorts, the number of mortality events was quite different between these two cohorts (table 2).

Despite the biological plausibility and statistical significance of this association between sLOXL2 levels and IPF outcomes, there remain significant limitations that should be considered. These observations were made using small IPF cohorts, which may result in imprecise point estimates. Although treatment assignment was forced into the Cox models, the risk estimates associated with sLOXL2 in the ARTEMIS-IPF analysis also remains subject to residual confounding effects of the study drug, which was associated with a 1.7-fold increase in risk for IPF disease progression [13]. Exclusion of $30 \%$ of the GAP subjects due to missing baseline lung function data may have also biased the results. Subjects with missing baseline lung function data may have had more advanced IPF disease, as reflected by more deaths (50\% versus $37 \%$ ) and a slightly higher risk for death (HR 1.75, 95\% CI 0.95-3.23; p=0.07). Finally, the low picogram levels of sLOXL2 require an assay of extremely high sensitivity. We expect that the CART-identified sLOXL2 thresholds may change when a more robust and standardised assay is developed and a more comprehensive IPF population has been surveyed.

Given the high likelihood that LOXL2 is involved in the pathogenesis of IPF, future studies should examine the diagnostic, prognostic and predictive potential of sLOXL2. It is possible that SLOXL2 may enhance the positive predictive value of current diagnostic criteria [15] for IPF. Future studies should also consider whether sLOXL2, along with other promising prognostic IPF biomarkers (e.g. matrix metalloproteinase (MMP)1, MMP7, KL-6, periostin, surfactant protein-A and D, CC chemokine ligand 18, vascular endothelial growth factor and YKL-40) [19-29], as well as prognostic scores [30, 31] and radiological modalities [32], might have prognostic value for helping physicians and patients anticipate the patient's IPF disease progression. This might include evaluation of serially collected sLOXL2 levels and their relationship to IPF acute exacerbations, which represent a terminal event for many IPF patients [33]. Finally, sLOXL2 levels might be predictive of an IPF patient's response to targeted therapy. Simtuzumab, a humanised monoclonal antibody that is an allosteric inhibitor of LOXL2, is being investigated in a phase 2 clinical trial (www.ClinicalTrials.gov identifier number NCT01769196) for treatment of IPF. This study will help to determine whether baseline sLOXL2 levels predict response to simtuzumab, as well as confirm our findings presented in this article that patients with high baseline LOXL2 levels are at increased risk for poor IPF outcomes.

In summary, these data demonstrate a novel association between higher sLOXL2 levels and increased risk for poor IPF outcomes. Given this association was demonstrated in two independent IPF populations and that LOXL2 has been shown to be a disease-driver of pathogenesis of fibrotic diseases in animal models, this association may be causal in nature. However, these results do not indicate that sLOXL2 is a suitable biomarker for IPF at this time. Future work should evaluate the epidemiology of sLOXL2 not only in patients with IPF, but all fibrotic lung diseases, which will be necessary to determine whether sLOXL2 has any diagnostic, prognostic, or predictive value in the management of IPF patients.

\section{References}

King TE Jr, Pardo A, Selman M. Idiopathic pulmonary fibrosis. Lancet 2011; 378: 1949-1961.

2 Raghu G, Striker LJ, Hudson LD, et al. Extracellular matrix in normal and fibrotic human lungs. Am Rev Respir Dis 1985; 131: 281-289.

3 Parra ER, Teodoro WR, Velosa AP, et al. Interstitial and vascular type V collagen morphologic disorganization in usual interstitial pneumonia. J Histochem Cytochem 2006; 54: 1315-1325.

4 Tzortzaki EG, Koutsopoulos AV, Dambaki KI, et al. Active remodeling in idiopathic interstitial pneumonias: evaluation of collagen types XII and XIV. J Histochem Cytochem 2006; 54: 693-700.

5 Suki B, Ito S, Stamenovic D, et al. Biomechanics of the lung parenchyma: critical roles of collagen and mechanical forces. J Appl Physiol 2005; 98: 1892-1899.

6 Hinz B. Mechanical aspects of lung fibrosis: a spotlight on the myofibroblast. Proc Am Thoracic Soc 2012; 9: 137-147.

7 Buscemi L, Ramonet D, Klingberg F, et al. The single-molecule mechanics of the latent TGF- $\beta 1$ complex. Curr Biol 2011; 21: 2046-2054.

8 Shi M, Zhu J, Wang R, et al. Latent TGF- $\beta$ structure and activation. Nature 2011; 474: 343-349.

9 Barry-Hamilton V, Spangler R, Marshall D, et al. Allosteric inhibition of lysyl oxidase-like-2 impedes the development of a pathologic microenvironment. Nat Med 2010; 16: 1009-1017.

10 Mehal WZ, Iredale J, Friedman SL. Scraping fibrosis: expressway to the core of fibrosis. Nat Med 2011; 17: 552-553. 
11 Chien JW, Shao L, Lyman SK, et al. Elevated baseline serum lysyl oxidase 2 (LOXL2) levels are associated with higher risk for idiopathic pulmonary fibrosis disease progression. Eur Respir J 2012; 40: Suppl. 56, 562s.

12 Chien JW, Richards T, Gibson KF, et al. High baseline serum lysyl oxidase like-2 (sLOXL2) levels are associated with higher risk for idiopathic pulmonary fibrosis (IPF) disease progression and mortality. Am J Respir Crit Care Med 2013; 187: A5716.

13 Raghu G, Behr J, Brown KK, et al. Treatment of idiopathic pulmonary fibrosis with ambrisentan, selective antagonist of the endothelin A receptor: a randomized trial. Ann Intern Med 2013; 158: 641-649.

14 Wells AU, Desai SR, Rubens MB, et al. Idiopathic pulmonary fibrosis: a composite physiologic index derived from disease extent observed by computed tomography. Am J Respir Crit Care Med 2003; 167: 962-969.

15 Raghu G, Collard HR, Egan JJ, et al. An official ATS/ERS/JRS/ALAT statement: idiopathic pulmonary fibrosis: evidence-based guidelines for diagnosis and management. Am J Respir Crit Care Med 2011; 183: 788-824.

16 Antweiler RC, Taylor HE. Evaluation of statistical treatments of left-censored environmental data using coincident uncensored data sets: I. summary statistics. Environ Sci Technol 2008; 42: 3732-3738.

17 Talal A, Lyman S, Mikels-Vigdal A, et al. Serum lysyl oxidase-like 2 (LOXL2) enzyme levels correlate with fibrosis stage at baseline and with reversibility of fibrosis in cirrhotic patients with chronic hepatitis B after treatment with tenofovir disoproxil fumarate (TDF). Hepatology 2012; 56S: 263A.

18 Adamkewicz JI, Lyman S, Barnes CN, et al. Utility of serum lysyl oxidase-like 2 (LOXL2) measurement for the noninvasive estimation of liver fibrosis in patients with chronic hepatitis C infection. Hepatology 2011; 54: 311A.

19 Rosas IO, Richards TJ, Konishi K, et al. MMP1 and MMP7 as potential peripheral blood biomarkers in idiopathic pulmonary fibrosis. PLoS Med 2008; 5: e93.

20 Richards TJ, Kaminski N, Baribaud F, et al. Peripheral blood proteins predict mortality in idiopathic pulmonary fibrosis. Am J Respir Crit Care Med 2012; 185: 67-76.

21 Yokoyama A, Kohno N, Hamada H, et al. Circulating KL-6 predicts the outcome of rapidly progressive idiopathic pulmonary fibrosis. Am J Respir Crit Care Med 1998; 158: 1680-1684.

22 Satoh $\mathrm{H}$, Kurishima K, Ishikawa $\mathrm{H}$, et al. Increased levels of KL-6 and subsequent mortality in patients with interstitial lung diseases. J Intern Med 2006; 260: 429-434.

23 Naik PK, Bozyk PD, Bentley JK, et al. Periostin promotes fibrosis and predicts progression in patients with idiopathic pulmonary fibrosis. Am J Physiol Lung Cell Mol Physiol 2012; 303: L1046-L1056.

24 Kinder BW, Brown KK, McCormack FX, et al. Serum surfactant protein-A is a strong predictor of early mortality in idiopathic pulmonary fibrosis. Chest 2009; 135: 1557-1563.

25 Takahashi H, Fujishima T, Koba H, et al. Serum surfactant proteins A and D as prognostic factors in idiopathic pulmonary fibrosis and their relationship to disease extent. Am J Respir Crit Care Med 2000; 162: 1109-1114.

26 Prasse A, Probst C, Bargagli E, et al. Serum CC-chemokine ligand 18 concentration predicts outcome in idiopathic pulmonary fibrosis. Am J Respir Crit Care Med 2009; 179: 717-723.

27 Ando M, Miyazaki E, Ito T, et al. Significance of serum vascular endothelial growth factor level in patients with idiopathic pulmonary fibrosis. Lung 2010; 188: 247-252.

28 Korthagen NM, van Moorsel CH, Barlo NP, et al. Serum and BALF YKL-40 levels are predictors of survival in idiopathic pulmonary fibrosis. Respir Med 2011; 105: 106-113.

29 Okamoto M, Hoshino T, Kitasato Y, et al. Periostin, a matrix protein, is a novel biomarker for idiopathic interstitial pneumonias. Eur Respir J 2011; 37: 1119-1127.

30 Ley B, Ryerson CJ, Vittinghoff E, et al. A multidimensional index and staging system for idiopathic pulmonary fibrosis. Ann Intern Med 2012; 156: 684-691.

31 Mura M, Porretta MA, Bargagli E, et al. Predicting survival in newly diagnosed idiopathic pulmonary fibrosis: a 3-year prospective study. Eur Respir J 2012; 40: 101-109.

32 Maldonado F, Moua T, Rajagopalan S, et al. Automated quantification of radiologic patterns predicts survival in idiopathic pulmonary fibrosis. Eur Respir J 2014; 43: 204-212.

33 Collard HR, Moore BB, Flaherty KR, et al. Acute exacerbations of idiopathic pulmonary fibrosis. Am J Respir Crit Care Med 2007; 176: 636-643. 